\title{
A SMART BIOCHIP WITH HAND-HELD ANALYZER FOR MONITORING SPECIFIC ENVIRONMENTAL BIOMARKERS
}

\author{
Jungyoup (Jay) Han ${ }^{1 *}$, Junhai Kai ${ }^{l}$, Se Hwan Lee ${ }^{l}$, Aniruddha Puntambekar ${ }^{1}$, \\ Ji-Youn Lim ${ }^{2}$, Donggeun Sul ${ }^{2}$, and Chong H. Ahn ${ }^{1,3}$ \\ ${ }^{1}$ Siloam Biosciences, Inc., Cincinnati, USA \\ ${ }^{2}$ Graduate School of Medicine, Korea University, Seoul, Republic of Korea \\ ${ }^{3}$ School of Electronics \& Computing Systems, University of Cincinnati, USA
}

\begin{abstract}
A new smart disposable biochip with a hand-held analyzer for monitoring specific environmental proteomic biomarkers has been designed, developed and fully characterized using polymer microfabrication technology and chemiluminescence immunoassay detection method. High sensitivity, small size, and low cost of the developed system are very suitable for exposure monitoring as a point-of-care (POC) platform. In this paper, Rho GDP inhibitor and triose phosphate isomerase as potential environmental biomarkers of di(2-ethylhexl) phthalate (DEHP) and human disease associate with DEHP were used for the developed biochip with the analyzer, demonstrating a POC platform. The smart biochip based analysis only requires $<\sim 30$ minutes, comparing to $4 \sim 5$ hours of a conventional ELISA.
\end{abstract}

\section{INTRODUCTION}

As the most commonly used phthalate ester and well-known endocrine-disruptors, di(2-ethylhexyl) phthalate (DEHP) is widely used in variety of consumer products, such as building products, car products, clothing, food packaging, children's products and some medical devices made of polyvinyl chloride. DEHP, an environmentally toxic substance has been reported to have cytotoxic, immunotoxic, genotoxic and reproductive toxic properties, and in long term feed toxicological studies it has been shown to be carcinogenic in mice and rats [1]

The development of biochip has evolved through rapid growth over the last decade and has shown numerous applications in a number of biochemical analysis operations such as DNA analysis, proteomics analysis and clinical analysis, [2,3]. A significant application area for this technology is clinical diagnostics. The detection of specific diseases is possible by incorporating biomarkers specific to such agents. The early, rapid, and sensitive point-of-care testing (POCT) of the disease state becomes a vital goal for clinical diagnoses. So the microfluidic chips based on MEMS (Micro Electro Mechanical Systems) has been introduced for biomarker measurements for disease analysis and prevention. Such devices have many preferable advantages such as low sample/reagent volume, short analysis time, less sample waste, cost effectiveness, compatible manufacture processes, and potential for making disposable devices.

The development of biochip for clinical analysis has rapidly evolved over the last decade. Initial biochip research has also been followed by the development of $\mu$ TAS (Micro total analysis system), which has shown numerous applications in biochemical analysis. The explosive development of biochip for $\mu \mathrm{TAS}$ has been motivated by its capabilities in miniaturizing size, reducing detection time, improving sensitivity, and providing ease-in-operation. Specifically, there has been a large demand for the development of fully integrated plastic microfluidic immunoassay chips, which are ideal solutions for point-of-care testing for monitoring human biomarkers level in disease analysis and prevention. Such devices have many favorable advantages such as low sample/reagent volume, short analysis time, less sample wastage, cost effectiveness, compatible manufacturing processes, and potential for making disposable devices.

The paper is organized as follows: first we introduce a protocol of the chemiluminescence-based sandwich immunoassay sequence in the biochip and design scheme of the developed biochip with solid propellant nitrogen gas generator. Next configuration of the developed hand-held analyzer and biochip are demonstrated. Then, result of the testing detection of biomarkers and operation of the developed hand-held analyzer with biochip is discussed.

\section{DESIGN AND FABIRCATION}

\section{Solid Phase Immunoassay on biochip}

Sandwich immunoassay is a kind of solid phase immunoassay applied for the determination of macromolecular antigens, e.g., protein and peptide, where simultaneous binding of two antibodies to the antigen is allowed without steric hindrance. The sample is incubated onto a solid phase, which is coated with an excess of affinity-purified anti-analyte (capture antibody) and blocked by high concentration dummy protein solution.

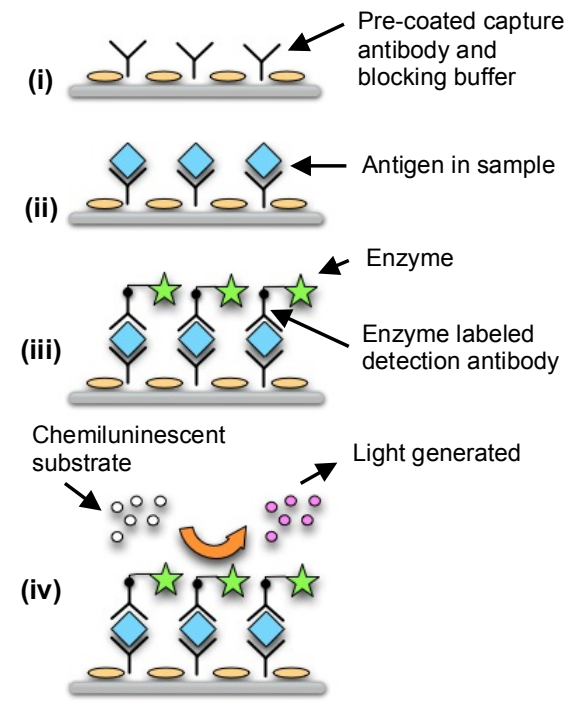

Figure 1: Protocol of the chemiluminescence-based sandwich immunoassay sequence in the biochip: (i) incubating capture anti-body and blocking; (ii) incubating target analyte; (iii) incubating enzyme labeled detection antibody; and (iv) catalyzing chemiluminescent substrate.

During the subsequent incubation, the capture antibody binds the analyte specifically. All other sample constituents are washed out and then bound analyte is quantified in a second step by adding an excess of labeled anti-analyte antibody (detection antibody). After incubation the unbound antibody is washed out and the signal from the solid-phase-bound detection antibody is directly related to the analyte concentration in the sample. For 
chemiluminescence immunoassay, the detection antibody is labeled with chemiluminescence enzyme that catalyzes chemiluminescence substrate to generate the light signal. Figure 1 shows the protocol of chemiluminescence based sandwich immunoassay.

Passive adsorption of protein follows typical saturation principles. In addition, the saturation will occur in molecular density in range of $10^{-9} \sim 10^{-8} \mathrm{~mol} / \mathrm{m}^{2}$ in most hydrophobic polymer surface depending on the protein molecular weight, solution condition, and surface condition. Increasing surface-volume ratio and shortening diffusion distance should decrease the incubation time of protein adsorption on the solid surface. That means the assay time for solid phase immunoassay will be significantly reduced by using microfluidic biochips.

\section{Microfluidic "Smart" Biochip}

Figure 2 illustrates the developed biochip for simplicity. The biochip is composed of six (6) microfluidic chambers for assay reagents and sample. The capture antibody is pre-conjugated onto the detection chamber. Four chambers are preloaded with reagent solution: detection antibody, $1^{\text {st }}$ washing buffer, $2^{\text {nd }}$ washing buffer and chemiluminescence substrate. A two-step buffer wash ensures that any residue of the HRP-labeled secondary antibody does not interact with the chemiluminescence substrate to produce a false signal. The empty "sample" chamber will be loaded with target sample before testing.

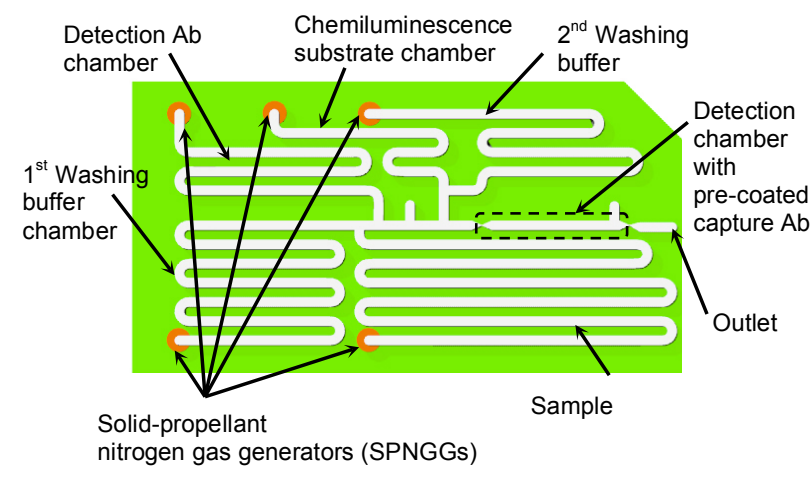

Figure 2: Schematic diagram of the developed "smart" biochip $(60 \mathrm{~mm} \times 30 \mathrm{~mm})$, which has microchannels for detection and pre-coated capture antibody, sample solution, washing buffers and substrate solution.

As shown in Figure 2, the biochip contains a detection chamber where the capture antibody was coated prior to assembly. After assembly, all the microfluidic channels in the biochip were coated with a blocking agent to minimize non-specific adsorption. Following this, all the reagents and buffer solutions were loaded using capillary sampling. Using selective surface modification techniques we can ensure that only the reagent and buffer chambers are hydrophilic whereas the remaining portions are strongly hydrophobic. The passive valves at the end of each chamber, and the air gap channels further ensure that the liquids only fill up the relevant chambers. Following this step, each of the reagent/buffer inlets in sealed to ensure that no liquid flows out when pressure is applied.

A key advantage of the developed biochip is the solid-propellant based microfluidic pump scheme [4]. This allows us to develop a fully integrated biochip that includes all the fluidic components equipped on-chip. Eliminating bulky, power-hungry components such as pumps affords size and cost benefits whereas eliminating fluidic interconnects to such components improves the chip reliability.
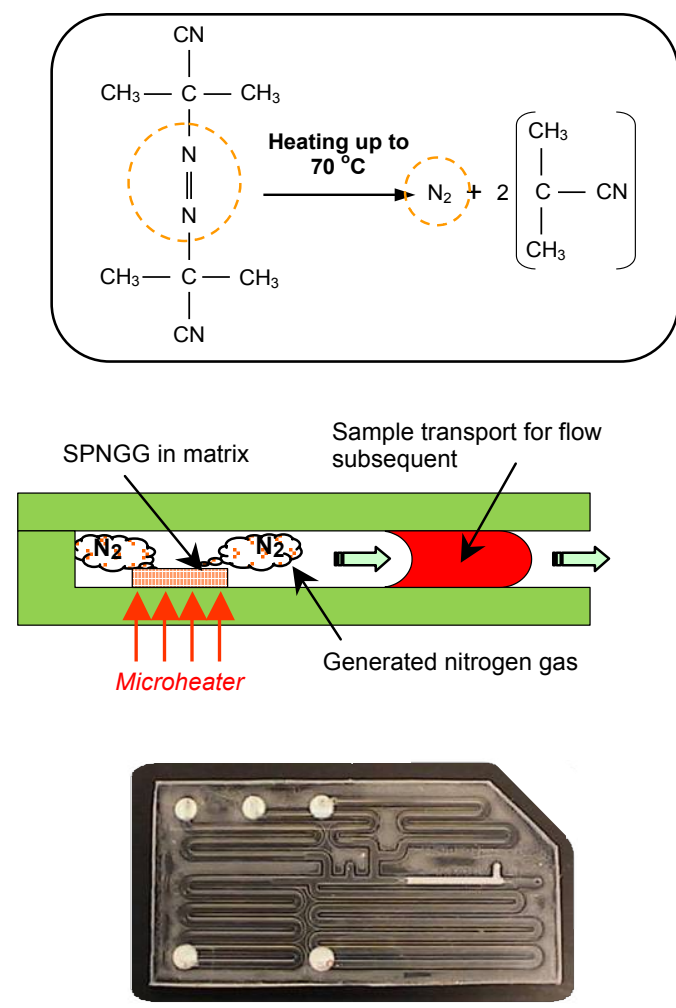

Figure 3: (a) Decomposition mechanism of the solid-propellant nitrogen gas generator (SPNGG); (b) schematic diagram of solid-propellant nitrogen gas generator (SPNGG) operation [4] and (c) photograph after assembly of biochip with SPNGGs (5 circular spots at the end of the chambers shown)

The functional pressure generator consists of a solid chemical propellant positioned on a resistive microheater. The solid propellant (AIBN: azobis-isobutyronitrile) decomposes at $70^{\circ} \mathrm{C}$ and releases non-toxic Nitrogen gas a by-product as shown in Figure 3(a). This device can be easily integrated with the biochip using standard lithography/screen-printing techniques. The amount of Nitrogen generated is governed by the applied thermal power and can be easily regulated. Due to its compact size, easy fabrication, easy integration, high reliability, biologically inert gas output, and functionality of gas generation, this pressure generator serves as an excellent driving mechanism for the biochip application

\section{Hand-held Analyzer}

We have developed a chemiluminescence based electronic analyzer which is capable of analyzing low-level light signal output from a dedicated biochip and controls the flow sequence of the biochip and captures the light signal from the biochip using an in-built photo detection circuitry and displays the captured data as a corresponding concentration value, shown in Figure 4 . The analyzer can be configured for use of a biochip towards both enzyme activity detection and protein biomarker concentration measurement using sandwich immunoassay protocol.

The analyzer is comprised of two major user interface controls: a) keypad and b) 16 character LCD display. In addition to the interface, the analyzer also incorporates a PS/2 data port 
with USB communication protocol. The analyzer can be connected to a personal computer (PC) and programmed using dedicated software installed on the computer.

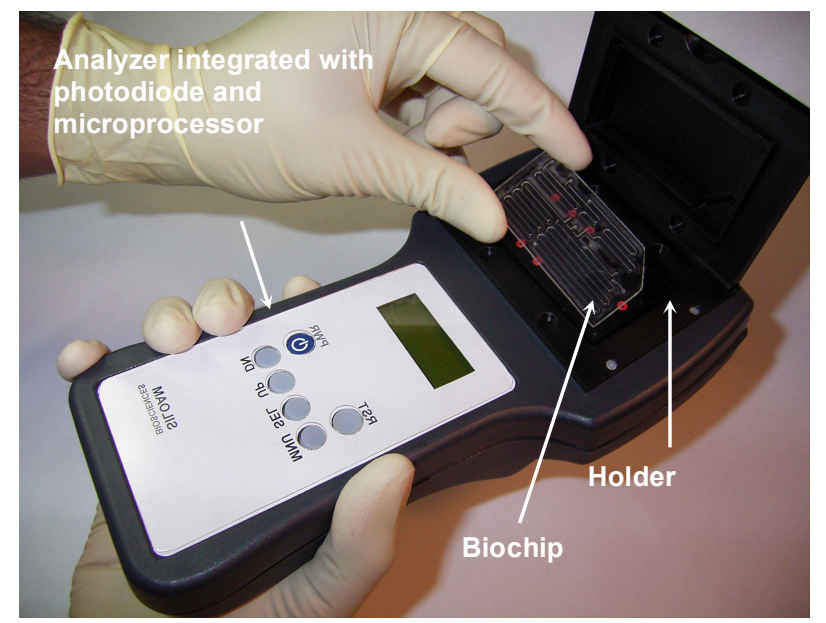

Figure 4: Developed hand-held analyzer integrated with photodiode and microprocessor

The microprocessor unit is built on the commercially available microprocessor PIC16F877A from Microchip Inc. The microprocessor is integrated with digital $\mathrm{I} / \mathrm{O}$ ports and 8 analog input channels. The system has two $5 \mathrm{~V}$ regulated power supplies: one supply is dedicated for microprocessor alone and the second $5 \mathrm{~V}$ regulated power supply is utilized for all the peripheral devices used along with the system. The microprocessor configured on the mother board controls the heater board which drives microheaters for operation of SPNGGs with pre-programmed software and receives light signal thru the optical detection module.

The holder was specially designed for tight light sealing and integration of five (5) microheaters underneath of SPNGGs on a biochip well aligned. Optical signal detection module is attached to the bottom of the holder with a hollow hole aligned with the detection chamber of the biochip.

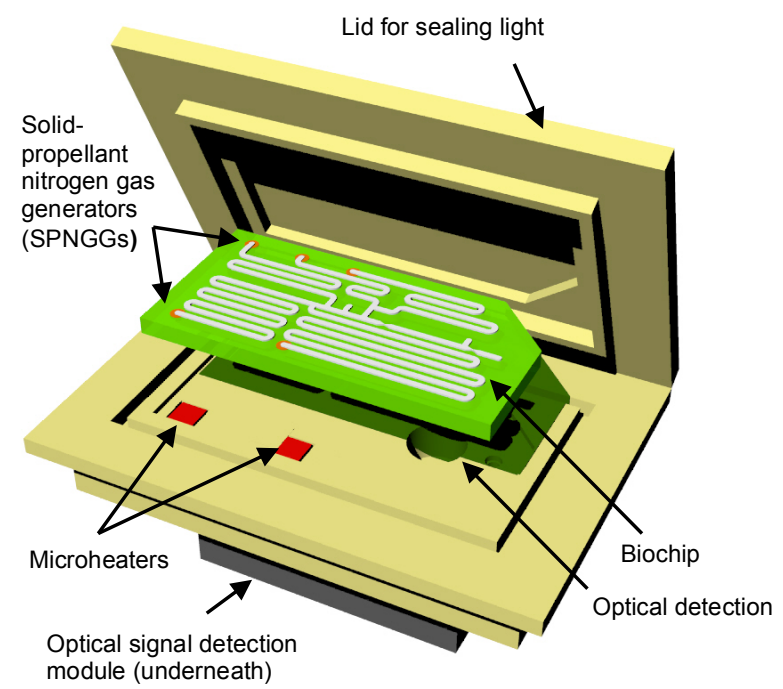

Figure 5. Schematic diagram of developed biochip, holder and optical signal detection module. Five (5) microheaters are deposited on the holder, which are aligned with solid-propellant nitrogen gas generators (SPNGGs) on the biochip.

\section{RESULT AND DISCUSSION}

We have developed the fully integrated biochip including solid-propellant nitrogen gas generators for evaluating the fluidic sequencing of the biochip with sandwich immunoassay. Microphotographs of the biochip are shown is Figure 6. In the paper we used five SPNGGs. After loading target analyte into the sample chamber, the first SPNGG fired to deliver target analyte to the detection chamber pre-coated with capture antibody. Then the second SPNGG to deliver the detection antibody into the detection chamber, pushing the target analyte incubated at the detection chamber for the previous step. With completion of second flow, the third SPNGG were used for delivery of the $1^{\text {st }}$ and $2^{\text {nd }}$ washing buffer, sequentially. After ignition of the fifth SPNGG for substrate delivery the analyzer read the optical signal developed from the detection chamber. Note that this image sequence uses color dyes for visualization.

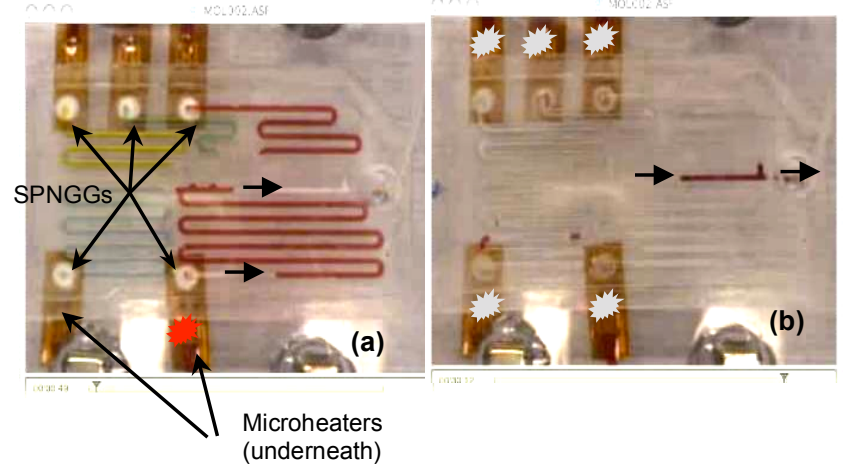

Figure 6: Operation of the smart biochip with five SPNGGs, microheaters underneath each SPNGG, and chambers: (a) the sample is delivered to detection chamber by the first SPNGG and (b) after completing all immunoassay sequences, the biochip is ready for detection of optical signal using the analyzer.

The SPNGG successfully delivered the reagents at controlled rates ( 22 minute) for completing on-chip assay. By modulating the nitrogen gas generation rate of SPNGG, we can optimize the flow rates and delivery times to ensure that the immunoassay sequence is completed satisfactorily. During optimization of the flow rate, there were significant "backflow" where the assay reagent entered a channel adjoining the detection chamber. For example, the sample partially entered the delivery channel for detection antibody and buffer/substrate. However, using the two-step wash sequence, any residue due to backflow can be pumped out before unwanted interactions.

Previously we have reported identification of toxicological biomarkers of DEHP [5]. Through the western blotting analysis for HepG2 secreted proteins, we have clearly shown three (3) up-regulated expressions of proteins, Rho-GDP initiator (Rho-GDI), gelsolin, and triose phosphate isomerase (TIM). A low concentration of DEHP led to up-regulation of Raf kinase inhibitory protein (RKIP) in HepG2 cells, while a high concentration of DEHP reduced the expression of RKIP. From this result we selected Rho GDP initiator and TIM as potential phthalate biomarkers.

As seen in Figure 7, the chemiluminescence -based sandwich immunoassay of selected proteins on the developed smart biochip 
with the hand-held analyzer was successfully evaluated $\left(>R^{2}=0.99\right.$ for both Rho-GDI and TIM).
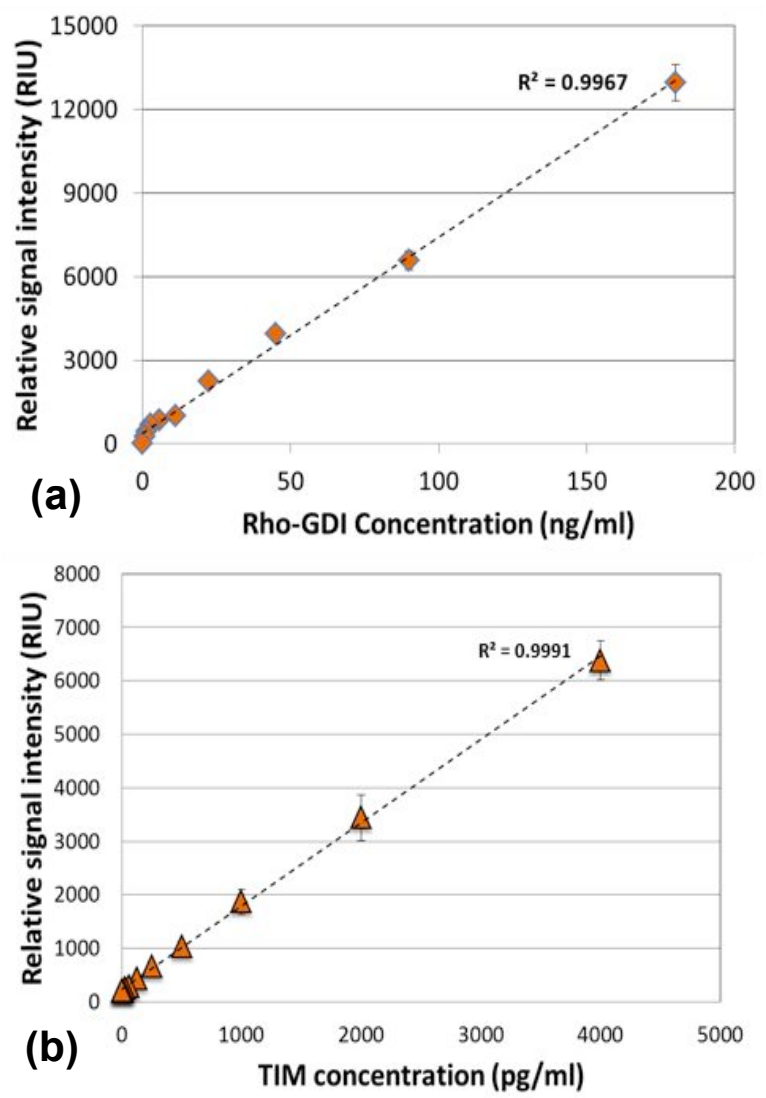

Figure 7: Immunoassay test results for concentrations of (a) Rho-GDI and (b) Triose phosphate isomerage (TIM) using developed smart biochip with hand-held analyzer. This clearly demonstrates the ability of the developed biochip and analyzer for high sensitivity detection of specific phthalate biomarkers.

In case of POCT application, a battery powered hand-held photodiode based optical analyzer was developed and characterized. By combining with the hand-help analyzer, the biochip exhibits powerful capabilities as the key sensing unit in terms of rapid and accurate point-of-care testing for clinical diagnostics. The features of high sensitivity and rapid analysis from the proposed system makes it uniquely well suited for POCT of specific environmental biomarkers for early warning of disease.

\section{ACKNOWLEDGEMENT}

This work was partially supported by the Ministry of Environment of South Korean Government as "The Eco-Technopia 21 project" (2009-09001-0075-0).

\section{REFERENCES}

[1] R Kavlock, D Barr, K Boekelheide, W Breslin, "NTP-CERHR expert panel update on the reproductive and developmental toxicity of di(2-ethylhexyl) phthalate," Reproductive Toxicology. 22, 291-399 (2006)

[2] C Malines, M Niggemann, and BD MacCraith, "Multi-analyte optical chemical sensor employing a plastic substrate," Measurement Science and Technology, 11(8) 1105-1110, (2000)
[3] R Ehrnstorm, "Miniaturization and integration: challenges and breakthrough in microfluidics," Lab on a Chip, 2, $26 \mathrm{~N}-30 \mathrm{~N}(2002)$

[4] J Han, S Lee, and C Ahn, "A disposable on-chip pressure actuator using IR-induced thermolysis for sample transport," MicroTAS 2006, Kyoto, Japan 521-523 (2006)

[5] S Choi, SY Park, J Jeong, E Cho, S Phark, M Lee, D Kwak, JY Lim, D Sul, "Identification of toxicological biomarkers of di(2-ethylhexyl) phthalate in proteins secreted by HepG2 cells using proteomic analysis," Proteomics, 10(9), 1831-46 (2010)

\section{CONTACT}

*J. Han, tel: +1-513-429-2976; jhan@siloambio.com 